\title{
Genome-wide Association Analysis of Red Flesh Character Based on Resequencing Approach in Peach
}

\author{
Huihui Liu, Ke Cao', Gengrui Zhu, Weichao Fang, Changwen Chen, Xinwei Wang, and \\ Lirong Wang ${ }^{1}$ \\ The Key Laboratory of Biology and Genetic Improvement of Horticultural Crops (Fruit Tree Breeding \\ Technology), Ministry of Agriculture, Zhengzhou Fruit Research Institute, Chinese Academy of \\ Agricultural Sciences, Zhengzhou 450009, China
}

\begin{abstract}
AdDitional INDEX words. anthocyanin, RNA-seq, single-nucleotide polymorphism, sugar transporter
Abstract. Anthocyanins are important molecules that are responsible for fruit color formation and are also beneficial to human health. To date, numerous structural and regulatory genes associated with anthocyanin biosynthesis in peach (Prunus persica) have been reported based on linkage analysis. In this study, we sought to identify further genes associated with anthocyanin content in peach by conducting a genome-wide association analysis of 129 peach accessions to detect markers associated with the trait. Significant association signals were detected when anthocyanin content was considered a qualitative character but not when it was considered a quantitative trait. We detected an association region located between 11.7 and $13.1 \mathrm{Mb}$ in chromosome 1 , a region in which only 133 of 146 genes have previously been functionally annotated. Gene ontology annotation of the genes in this region showed that membraneassociated genes (including one gene encoding a chloride channel protein and 17 sugar transport/carrier-associated genes) were significantly enriched, and we focused on these in subsequent analyses. Based on in vitro induction of anthocyanins in fruit flesh using different exogenously applied sugars and subsequent culture, we found that the expression level of 3 of the 18 membrane-associated genes, Prupe.1G156300, Prupe.1G156900, and Prupe.1G157000, increased during induction treatment. Furthermore, during the fruit development period of a white-fleshed and a redfleshed peach cultivar, the expression of one gene encoding a transmembrane sugar transport protein was observed to be positively correlated with anthocyanin biosynthesis. These results will facilitate understanding of the molecular mechanism of anthocyanin biosynthesis in peach.
\end{abstract}

Anthocyanins are important pigments in plants. These compounds are found in stems, leaves, petals, and flesh of fruit (Forkmann and Martens, 2001; Gould et al., 2009), and play important roles in attracting insect pollinators and animal seed dispersers, and in disease and insect resistance, like fungi, herbivores, cold, and excess radiation (Chalker, 2002, 2010; Karageorgou and Manetas, 2006). For example, anthocyanins can absorb visible light without participating in photosynthesis; their presence in leaves should reduce the probability of photon capture by chlorophylls, thereby lowering photosynthesis (Karageorgou and Manetas, 2006). Anthocyanins also are beneficial to human health, and it has been found that foods with high anthocyanin content reduce free radicals, promote blood circulation, prevent cardiovascular and cerebrovascular diseases, and also have antiaging and anticancer properties (Chen et al., 2006; Choi et al., 1997; Xia et al., 2010). Yang et al. (2018) found that anthocyanins from purple wheat

Received for publication 18 Dec. 2018. Accepted for publication 17 Apr. 2019. This work was supported by the National Natural Science Foundation of China (grant no. 31672130) and the Agricultural Science and Technology Innovation Program (grant CAAS-ASTIP-2017-ZFRI-01). We thank Central Public-interest Scientific Institution Basal Research Fund the Preliminary Establishment and Capacity Improvements of the Molecular Breeding Platform for Fruit Trees (Y2019PT19-02).

The authors declare that they have no competing interests.

The RNA-sequencing data of two cultivars (Tianjin Shui Mi and Hakuho peach) have been deposited into the Figshare database (https://doi.org/10.6084/m9. figshare.5576368.v1)

1Corresponding authors. Email: wyandck@126.com or wanglirong@caas.cn.
(Triticum aestivum) can inhibit the infection capacity and proliferation of hgc- 27 and mgc-803 gastric cancer cells. Lila et al. (2016) argued that anthocyanins undergo enterohepatic recycling in the human body, leading to prolonged residence time, and thus enhanced bioavailability; however, levels were found to be very low in routine blood samples taken after ingestion. Single oral administration of anthocyanin has been demonstrated to reduce smoking-induced endothelial dysfunction in young healthy smokers (Akane et al., 2018), whereas cyanidin-3-O-glucoside also can alleviate the $\mathrm{Pb}$-induced decrease in progesterone biosynthesis to reduce the toxicity of heavy metals in rat Leydig cells, and anthocyanin is able to chelate heavy metals and reduce metal ion concentrations, thereby further alleviating heavy metal-induced toxicity (Wen et al., 2018; Zhou et al., 2017).

Peach, a member of the Rosaceae family native to China, is among the four most widely cultivated fruit tree species worldwide (Tuan et al., 2015), and serves as a model for genetic studies owing to its small genome size of $265 \mathrm{Mb}$ (Dirlewanger et al., 2004; Shulaev et al., 2008; Zhu et al., 2012), and the whole-genome sequence of peach has been released (Verde et al., 2013), providing an important foundation for the genome-wide identification of genes in this species (Verde et al., 2017). Typically, peach has one of two types of flesh color: white or yellow. After studying the anthocyanin pigments in peaches, research found that there were two main pigments in peach, cyanidin-3-glucoside and cyanidin-3rutinoside, with the former content being $\approx 10$ times more than the latter. In addition, no clear differences were observed 
in pigment contents of white and yellow flesh accession (Tomas-Barberan et al., 2001). In recent decades, peaches with red flesh have attracted the attention of a large number of researchers worldwide (Ding, 2017).

To date, candidate genes involved in biosynthesis, regulation, and response to induction by environmental stimuli, such as light, and temperature, and sugar and plant hormones, have been identified in peach (Donoso et al., 2016; Eduardo et al., 2011; Frett et al., 2014; Jaakola, 2013; Jiao et al., 2014; Rahim et al., 2014; Zhou et al., 2017). Among the biosynthetic genes identified, there are a number of structural genes that directly encode enzymes required for the biosynthesis of anthocyanin, including chalcone synthase (CHS), chalcone isomerase, leucoanthocyanidin dioxygenase/anthocyanidin synthase, and UDP-glucose flavonoid 3-O-glucosyltransferase (UFGT) (Tsukaya, 2004). It is currently believed that the process of anthocyanin biosynthesis is mainly regulated by three classes of transcription factors, namely, v-myb avian myeloblastosis viral oncogene homolog (MYB), basic helix-loop-helix (bHLH), and tryptophan-aspartic acid 40 (WD40) transcription factors (Xu et al., 2015). Among these, MYB transcription factor genes often are found to be the major determinant of anthocyanin accumulation by acting in conjunction with bHLH and WD40 proteins to activate key anthocyanin biosynthetic genes (Allan et al., 2008; Lin-Wang et al., 2010; Petroni and Tonelli, 2011). The major transcription factor R2R3 MYB10/MYB1/MYBA was shown to be associated with activation of the anthocyanin biosynthesis pathway in the Rosaceae (Allan et al., 2008; LinWang et al., 2010), leading to the development of red flesh pigmentation, and is located within the interval of the major markers associated with blush on linkage group 3 (Frett et al., 2014). However, Zhou et al. (2015) found that the sequence of PpMYB10.1 did not segregate along with the red flesh phenotype, although their team identified another gene, a NAC transcription factor gene $(P p B L)$, that was significantly differentially expressed between the red- and white-fleshed cultivars and confirmed that this gene could affect anthocyanin biosynthesis by regulating PpMYB10.1 using transient transformation of tobacco (Nicotiana benthamiana) and peach (Zhou et al., 2015). Recently, Cao et al. (2018) identified 66 genes were significantly correlated with anthocyanin contents through RNA-sequencing (RNA-seq), 22 of which previously reported as regulatory, biosynthetic, and transporter genes, including $P p M Y B$ and $P p B L$, are involved in the regulatory network of anthocyanins. Unfortunately, no polymorphic sites have been identified in the coding sequence of the genes in red-fleshed peach. More research is needed to locate key genes involved in red flesh traits in peach.

Genome-wide association study (GWAS) has proved to be a useful tool for dissecting the genetic locus of complex agronomic characters in arabidopsis (Arabidopsis thaliana), rice (Oryza sativa), maize (Zea mays), wheat, and barley (Hordeum vulgare) (Gyawali et al., 2017; Kim et al., 2016; Revilla et al., 2016; Togninalli et al., 2018; Turuspekov et al., 2017). It has the advantage of not requiring prior establishment of a mapping population and can detect multiple allele loci, thereby saving both time and labor. In peach, Cao et al. (2012) demonstrated GWAS analysis to be an effective complement to traditional quantitative trait locus mapping.

In this study, we performed a GWAS with the aim of locating the region associated with anthocyanin content in peach flesh. Candidate genes were identified based on an in vitro experiment involving sugar induction of fruit flesh and comparing these genes at different stages of fruit development. The findings of this study provide a more comprehensive understanding of the molecular mechanisms underlying anthocyanin biosynthesis and could be useful in development and application of molecular markers for peach breeding.

\section{Materials and Methods}

Plant materials. Three sources of plant materials were used in this study, the first of which was 129 peach accessions previously used in GWASs (Cao et al., 2016; Supplemental Table 1). For each of these cultivars, fresh samples of the mesocarp of three ripe fruit were mixed and frozen in liquid nitrogen and then stored at $-80^{\circ} \mathrm{C}$ for anthocyanin evaluation. In addition, total DNA was extracted from the fresh leaves of these accessions for genome re-sequencing.

The second source of material was the flesh of 'Tianjin Shui Mi' peach fruit harvested at $15 \mathrm{~d}$ before fruit maturity (the key stage of anthocyanin accumulation, $80 \mathrm{~d}$ after blooming). The flesh of the harvested fruit was cultured in the presence of exogenous sugar for candidate gene screening. The ripe stage was evaluated according to the grading standards described by Zhang (2008).

The third source of material was two peach accessions with different anthocyanin contents, a white-fleshed peach ('Hakuho') and a red-fleshed peach ('Tianjin Shui Mi'), in which anthocyanin accumulates during the late stage of fruit development (Ding, 2017). The maturation periods of these accessions are all early July in Zhengzhou, China. Three fruit of each cultivar were harvested at three stages, from which three replicate fresh samples were analyzed separately.

Anthocyanin Quantification in FRUit Flesh. We used a high-performance liquid chromatography system (HPLC; Shimadzu Corp., Kyoto, Japan) coupled with photodiode array detector to analyze flesh anthocyanin contents $1 \mathrm{~cm}$ depth from surface of 129 peach accessions at mature time, which are characterized by different flesh colors, and also in two accessions during different fruit development periods, and in sugarinduced fruit with different treated times. Accurately weighed samples $(1 \mathrm{~g})$ of powdered fruit flesh were placed into $50-\mathrm{mL}$ flasks containing $25 \mathrm{~mL}$ of a methanol/formic acid/water $(45: 1: 4 \mathrm{v} / \mathrm{v} / \mathrm{v})$ mixture and incubated overnight $(12 \mathrm{~h})$ at $4{ }^{\circ} \mathrm{C}$. Following centrifugation at $2000 g_{\mathrm{n}}$, the supernatant was collected and used for HPLC analysis to evaluate cyanidin-3glucoside and cyanidin-3-rutinoside. The injection volume was $20 \mu \mathrm{L}$, the flow rate was $1 \mathrm{~mL} \cdot \mathrm{min}^{-1}$, and the detection wavelengths were 280 and $516 \mathrm{~nm}$. A standard curve was prepared using cyanidin-3-O-glucoside as a standard sample. In addition, anthocyanin content was phenotyped in 2014 and 2015 as a qualitative character based on previously published plant genetic resources' evaluation criteria (Wang and Zhu, 2005). In the book, red pigment can be classified into two types according to some reference cultivars.

GENOME-WIDE ASSOCIATION STUDY. Three models of Tassel v4.0 software (Bradbury et al., 2007) were used to perform GWAS based on the previously identified single-nucleotide polymorphisms (SNPs) of 129 peach accessions (Cao et al., 2016). The first model was general linear model (GLM) without any consideration for principal component analysis (GLM-no PCA); the second was GLM but took PCA results into account as the fixed effect (GLM-PCA); and the third was a mixed linear 
Table 1. Details of primers used for quantitative real-time polymerase chain reaction to amplify genes related to sugar metabolism in peach fruit flesh.

\begin{tabular}{|c|c|c|c|}
\hline $\begin{array}{l}\text { Gene accession in reference } \\
\text { genome } v 1.0 / 2.0^{z}\end{array}$ & Forward primer $\left(5^{\prime}-3^{\prime}\right)$ & Reverse primer $\left(5^{\prime}-3^{\prime}\right)$ & $\begin{array}{c}\text { Product } \\
\text { (base pairs) }\end{array}$ \\
\hline ppa004132m/Prupe.1G156300 & GCCCTTTTGGTGGTGGAAAC & TAGGACTGGCGCGTAAAACA & 273 \\
\hline ppa004439m/Prupe. $1 G 156900$ & AGGTTGCCATTTCTGGAGGT & AATGCAAGGTCATGAAACGCAG & 286 \\
\hline
\end{tabular}

${ }^{\mathrm{z}}$ Peach reference genome v1.0 (Verde et al., 2013) and v2.0 (Verde et al., 2017).

model (MLM) using PCA results and the kinship as correction for population structure. In the model, a kinship matrix was calculated using Tassel software and the first three components of PCA were determined using genome-wide complex trait analysis (GCTA) (Yang et al., 2011). Manhattan and quantilequantile plots were generated using the qqman package in $\mathrm{R}$ (Turner, 2014), according to the association results for anthocyanin. We defined a whole-genome significance cutoff from the Bonferroni test threshold (significant genome-wide threshold: $-\log _{10} P=8.49$, calculated by dividing $0.01 \mathrm{using}$ $3,076,604$ SNPs). Gene ontology (GO) annotations of candidate genes were performed using Blast2GO (Conesa and Gotz, 2008) and plotted using WEGO (Web Gene Ontology Annotation Plot) (Ye et al., 2006).

IN VITRO INDUCTION OF ANTHOCYANIN SYNTHESIS BY SUGAR. A red-fleshed peach 'Tianjin Shui Mi' was selected to conduct this experiment. The flesh was cut into small pieces $(2 \times 2 \times$ $2 \mathrm{~mm}$ ) during the stage of development at which the flesh color changes $(\approx 15 \mathrm{~d}$ before the date of maturity). These samples were incubated in 2-(N-morpholino)ethanesulfonic acid (MES) culture medium ( $\mathrm{pH}=6.5)$, containing 100 mM MES ( $\mathrm{pH} 5.5$ ),
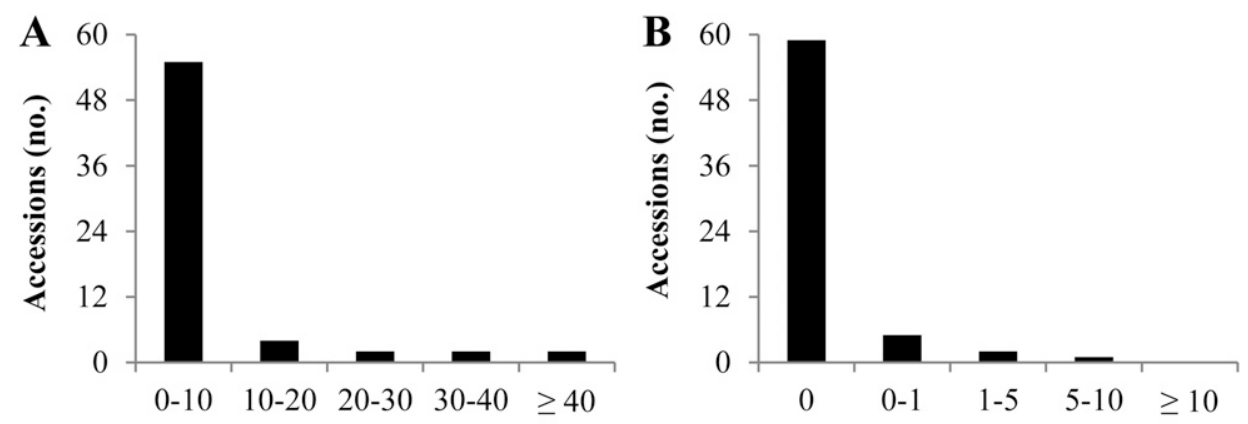

Cyanidin-3-glucoside content $\left(\mathrm{mg} \cdot \mathrm{kg}^{-1} \mathrm{FW}\right)$

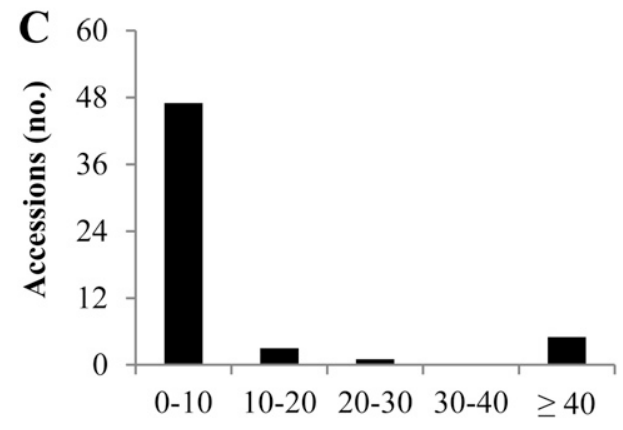

Cyanidin-3-glucoside content $\left(\mathrm{mg} \cdot \mathrm{kg}^{-1} \mathrm{FW}\right)$

Fig. 1. Anthocyanin quantification in fruit of 129 peach accessions in (A and B) 2014, (C) in 2015, and (D) classification. $\mathrm{FW}=$ fresh weight.
$5 \mathrm{~mm} \mathrm{CaCl}_{2}, 10 \mathrm{~mm} \mathrm{MgCl} 2,1 \mathrm{~mm}$ EDTA, $10 \mathrm{~mm}$ vitamin $\mathrm{C}$, and $100 \mathrm{~mm}$ sugar (glucose, sucrose, fructose, or sorbitol), at room temperature $\left(25^{\circ} \mathrm{C}\right)$. The incubation method was consulted from a previous study (Beruter and Studer Feusi, 1995; Do and Cormier, 1990; Jia, 2013) and adjusted a little. Light was supplied by fluorescent lamps with $62.5 \mu \mathrm{mol} \cdot \mathrm{m}^{-2} \cdot \mathrm{s}^{-1}$ for $24 \mathrm{~h}$. As a control, flesh was incubated in each MES medium containing mannitol to balance the osmotic potential. After treatment for 0,12 , and $24 \mathrm{~h}$, flesh samples were frozen in liquid nitrogen and stored at $-80{ }^{\circ} \mathrm{C}$ for subsequent RNA extraction and anthocyanin evaluation.

Transcriptional analysis OF FrUit Flesh INDUCED by SUGAR. Total RNA $(2 \mu \mathrm{g})$ was extracted of the induced fruit flesh using a Quick RNA Isolation Kit (Waryong, Beijing, China) according to the manufacturer's instructions. Purification of poly (A) messenger RNAs (mRNAs) was performed using oligo-dT attached to magnetic beads. The purified mRNAs were fragmented using ultra-sonication, and then subjected to first- and second-strand complementary DNA (cDNA) synthesis using random hexamer primers. Thereafter, cDNA fragments of $\approx 350$ base pairs $(b p)$ in size were gelpurified and used as templates in a polymerase chain reaction (PCR) and sequenced using a highthroughput sequencing platform (BGISEQ-500; GitHub, Shenzhen, China). Finally, the sequencing data were filtered using SOAPnuke (GitHub, 2016) and aligned against the peach reference genome v2.0 (Verde et al., 2017) for better annotation comparison of v1.0. Gene expression was calculated in terms of reads per kilobase per million mapped reads using Cufflinks v2.1.1 (Trapnell et al., 2010).

Real-time quantitative PCR. Fruit of the third source of material was used for extraction of RNA using an RNA extraction kit (EASYspin Plus; Aidlab, Beijing, China), and its purity was evaluated using a spectrophotometer (NanoDrop 1000; Thermo Fisher Scientific, Waltham, MA). cDNA was synthesized from $2 \mu \mathrm{g}$ of RNA using a First Strand cDNA Synthesis Kit (ReverTra Ace- $\alpha$-FSK101; Takara, Dalian, China) according to the manufacturer's protocol. According to gene sequences annotated by Verde et al. (2017), specific 
primers for three genes (Table 1) were designed using PrimerBLAST software (National Center for Biotechnology Information, Bethesda, MD).

Real-time quantitative PCR reactions were performed using the fluorescent quantitative PCR instrument (LightCycler 480; Roche Diagnostics, Mannheim, Germany) with a $10-\mu \mathrm{L}$ reaction mixture containing $10 \mathrm{ng}$ cDNA, $0.5 \mathrm{~mm}$ of each primer, $5 \mu \mathrm{L}$ Premix Taq solution (Takara), and $3.5 \mu \mathrm{L}$ double distilled water. The real-time quantitative PCR reaction program used was as follows: $95^{\circ} \mathrm{C}$ for $5 \mathrm{~min}$; followed by 35 cycles of $94^{\circ} \mathrm{C}$ for $30 \mathrm{~s}$, the annealing temperature for $30 \mathrm{~s}$, and $72^{\circ} \mathrm{C}$ for $30 \mathrm{~s}$; with a final elongation for $10 \mathrm{~min}$ at $72{ }^{\circ} \mathrm{C}$. Relative expression levels were estimated using the comparative $\mathrm{Ct}$ method (Livak and Schmittgen, 2001). The sequences of the primers used in this study are shown in Table 1 .

\section{Results}

Anthocyanin Phenotyping. The anthocyanin contents of 129 peach cultivars in 2014 (Fig. 1A and B) revealed cyanidin3-glucoside but cyanidin-3-rutinoside as the primary component of anthocyanin, therefore we focused on this molecule in
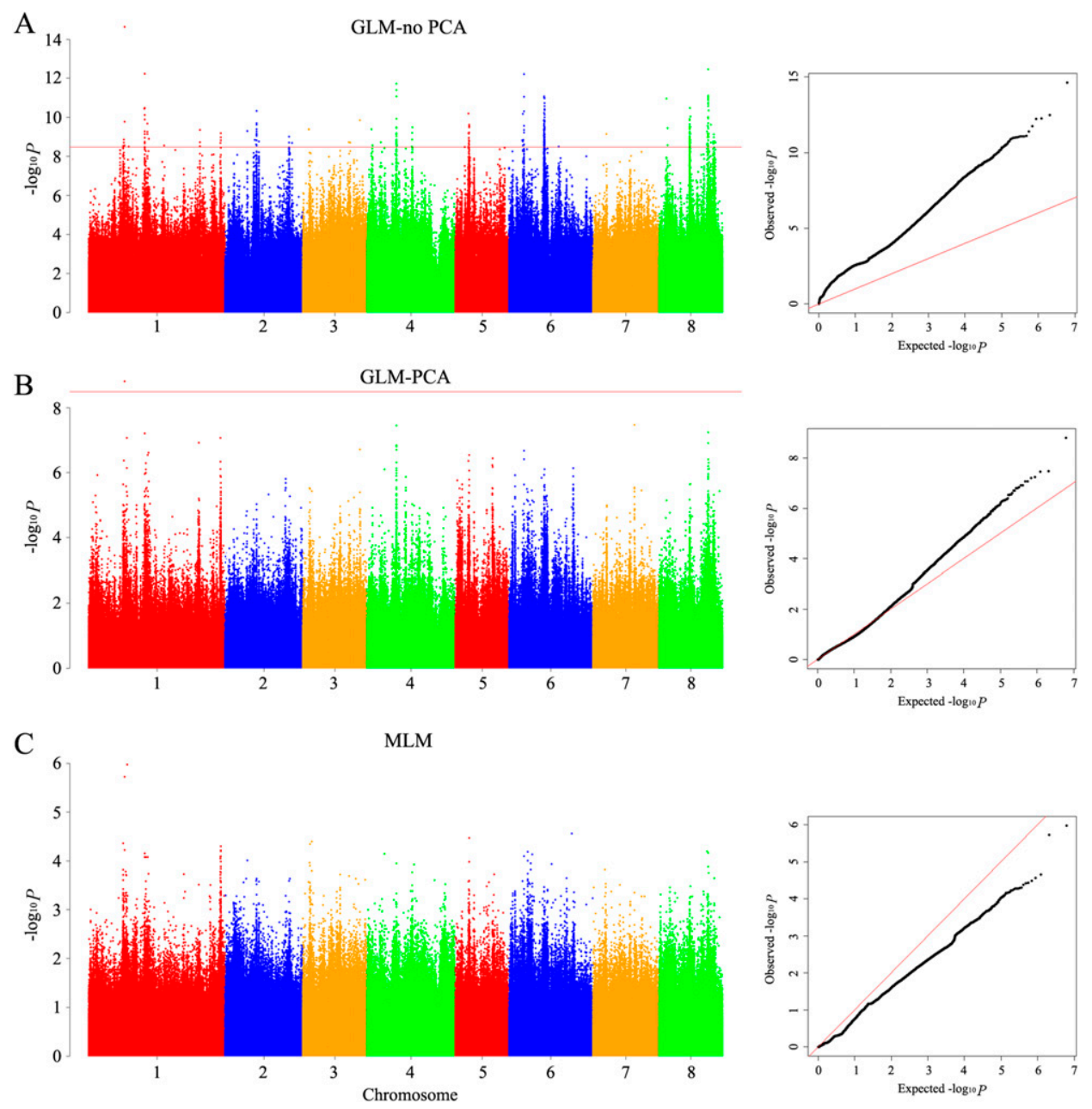

Fig. 2. Manhattan and quantile-quantile plots of estimated $-\log _{10} P$ from association analysis of anthocyanin absent/present in fruit of 129 peach accessions using (A) general linear model (GLM)-no principal components analysis (PCA), (B) GLM-PCA, and (C) mixed linear model (MLM). Negative $\log _{10} P$ values from the genomewide scan were plotted against single-nucleotide polymorphism position on each of the eight chromosomes. The dotted horizontal line indicates the genome-wide significance threshold $\left(-\log _{10} P=8.49\right)$. the subsequent analyses in 2014 and 2015 (Fig. 1A and C). Forty-eight of the assessed cultivars were found to be lacking in all anthocyanin evaluation, whereas among the remainder, cyanidin-3-glucoside showed skewed distribution (Fig. 1A and $\mathrm{C}$ ). Most of these latter accessions had anthocyanin concentrations ranging between 0 and $10 \mathrm{mg} \cdot \mathrm{kg}^{-1}$ of fresh fruit, whereas only $\approx 10$ cultivars showed higher concentrations. However, we detected no segregation distortion when the anthocyanin concentrations trait was considered as a qualitative haracter by direct observation (Fig. 1D).

Sequencing and GWAS. In the study, we identified 4,062,178 SNPs in 129 peach cultivars. After filtering the data using a minor allele frequency threshold of $\leq 0.05$ and absent in more than $20 \%$ assessed cultivars, finally, a total of 3,076,604 NPs remained for GWAS

Using the MLM, we performed association analysis of cyanidin-3-glucosidase in 2014 and 2015 (Supplemental Fig. 1A and because that model was proved to be suitable for the quantitative character (Korte and Farlow, 2013). The result showed that many association signals exceeded the genome-wide significance threshold $\left(-\log _{10} P>8.49\right.$ ) but presented a scattered distribution without any clear association peaks. This abnormal association may be related to the substantial bias in separation of the trait (Fig. 1A and C). In 2015, peak signals were found in $37.15,8.68$, $0.04,19.89,3.08,10.17,3.08$, and $5.25 \mathrm{Mb}$ of chromosome (Chr.) 1 to Chr. 8, respectively. And in 2014, peak signals were found in 36.51 , 4.94, 3.28, 15.54, 2.01, 19.84, 4.93, and $4.12 \mathrm{Mb}$ of Chr. 1 to 8 . The results indicated that there were no yearstable association regions of cyanidin-3-glucosidase contents in peach flesh considering the linkage disequilibrium decay of peach [about $20 \mathrm{~kb}$ (Cao et al., 2016)]. Furthermore, we found that significant SNPs were far from the candidate genes involved in anthocyanin biosynthesis. For example, a CHS gene, Prupe.1G002900, was located in $0.3 \mathrm{Mb}$ in $\mathrm{Chr}$. 1. A UFGT gene, Prupe.2G324700, was located in $30.0 \mathrm{Mb}$ in Chr. 2. A $M Y B$ gene, Prupe.3G163100, was located in $18.2 \mathrm{Mb}$ in Chr. 3.

When anthocyanin content was considered as a qualitative character (anthocyanin absent/present), three models were used for association analysis (Fig. 2A-C). The QQ-plot result showed that the GWAS of GLM-PCA present a well-defined distribution and the model could obtain clean and obvious associations. The false-positive association signals were avoided by controlling population structure comparing with the GLM-no PCA model (Fig. 2A). However, MLMs present an excessive regulation because no continuous 
and obvious association signals were found to be higher than the genome-wide significance level (Fig. 2C). Therefore, the most prominent association signal (Chr. 1: 12,265,694 bp, $-\log _{10} P=8.81$ ) being located in the interval between 11.7 and $13.1 \mathrm{Mb}$ on Chr. 1 of GLM-PCA (Fig. 2B) was selected for following analysis. To date, only 133 of 146 genes in this region have been functionally annotated, among which membrane-associated genes are significantly enriched (Fig. 3), including one gene encoding a chloride channel (CLC) protein, and 17 involved in sugar transport and galactosyltransferase activity (Table 2).

Candidate Gene identification USING FRUit FLeSh CULTURED WITH SUGAR. In the present study, expression of 17 enriched genes associated with sugar identified through GWAS was also evaluated in fruit flesh incubated with different sugars. Our results showed that with an increase in the duration of the culture period, anthocyanin was induced by glucose, sucrose, and fructose, whereas treatment with sorbitol had minor effects comparable to those of the control mannitol (Fig. 4A). In fruit subjected to sugar induction for $12 \mathrm{~h}$, we observed that glucose treatment resulted in the highest content of anthocyanin, followed by sucrose, whereas the levels of anthocyanin in flesh induced by fructose and sorbitol were lower than those induced by the mannitol control. In contrast, after $24 \mathrm{~h}$ of induction, the anthocyanin contents of fruit in all four sugar treatments were higher than those induced by mannitol, with the contents induced by glucose and sucrose being slightly higher than those induced by fructose.

On conducting RNA-seq analysis to assess the expression of the aforementioned 17 genes (Fig. 4B), we found that 13 showed no detectable expression. Among the remaining four genes, three (Prupe.1G56300, Prupe.1G56900, and Prupe.1G15700, corresponding to ppa004132m, ppa004439m, ppa023527m, respectively, annotated in reference genome v1.0) showed an expression pattern consistent with anthocyanin

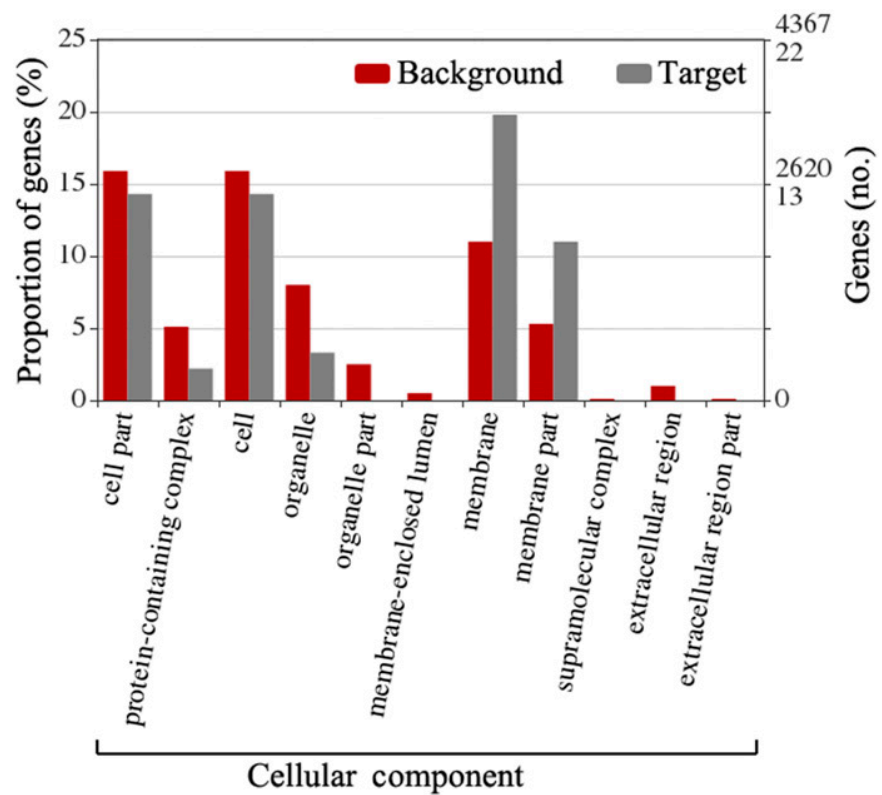

Fig. 3. The cellular component of gene ontology annotation of the 133 target genes and in comparison of all genes annotated in the genome (designated as background genes). The left and right y-axes present the proportion and number of genes among of different categories. induced by sugar, although the expression levels were higher at $12 \mathrm{~h}$ than at $24 \mathrm{~h}$. The expression level of the fourth of these genes (Prupe.1G166200) decreased with increasing culture time. Of the three genes with increased expression, Prupe.1G156300 had higher expression than Prupe.1G156900 and Prupe. 1 G157000.

CANDIDATE GENE IDENTIFICATION USING FRUIT FLESH DURING FRUIT DEVELOPMENTS. We further analyzed the expression of Prupe.1G56300, Prupe.1G56900, and Prupe.1G15700 in fruit flesh during different periods of fruit development (Fig. 5), and accordingly observed the steady expression of Prupe.1G156300 during the course of fruit development in the white-fleshed peach and induction at the mature stage in the red-fleshed peach, a pattern similar to the accumulation of anthocyanin. Prupe.1G157000 showed higher expression in the white-fleshed peach at the mature stage, in contrast to the observed anthocyanin levels. We were unable to detect the expression of Prupe.1G156900 in any of the two examined peach cultivars.

\section{Discussion}

GWAS is increasingly being applied in biological studies to facilitate direct correlation between phenotypic traits and the associated genetic mechanisms. The key genes for the red flesh phenotype of peach have previously been identified as PpMYB10.1 at $12.87 \mathrm{Mb}$ on Chr. 3 and $P p B L$ at 4.2 to 4.5 Mb on Chr. 4 (Zhou et al., 2015). A $D B F$ gene, known to control flesh color, has been located at the top of linkage group 5 (0.59$0.61 \mathrm{Mb}$ ) in a hybrid population of the blood peach cultivar $\mathrm{Wu}$ Yue Xian (Shen et al., 2013). Furthermore, DBF2, which has been determined to be responsible for red flesh color in almond (Prunus dulcis), was found to map to the end of linkage group 1 (corresponding to $35.2 \mathrm{Mb}$ in Chr. 1) in a cross between the almond cultivar Texas and the peach cultivar Earlygold (Donoso et al., 2016). During the study of our laboratory, 66 genes were identified through RNA-seq using different development of fruit of 'Tianjin Shui Mi' peach. Among of them, 13 genes were located at Chr. 1 and only ppa011068m (corresponding to $10.3 \mathrm{Mb}$ in $\mathrm{Chr}$. 1). In the present study, we conducted a GWAS to identify the marker associated with anthocyanin content using resequencing data. We found an obvious association signal with the GLM-PCA model and the association region to be located between 11.7 and $13.1 \mathrm{Mb}$ on Chr. 1 (Fig. 2C). This locus is close to that located in an interspecific cross (Prunus davidiana clone P1908 and P. persica cv. Summergrand) (Quilot et al., 2004), which is linked to the marker CFF14 (corresponding to $9.16 \mathrm{Mb}$ in Chr. 1) and gene ppa011068m (Cao et al., 2018). The different location from that described previously for the $D B F 2$ locus may be related to the different backgrounds.

Among the genes previously annotated in the putative association region, one gene encodes a CLC protein, and a further 17 are involved in sugar transport and galactosyltransferase activity. In arabidopsis (Wei et al., 2013) and rice (Diedhiou and Golldack, 2006), CLCs have been reported to be involved in plant adaptation to salt stress by regulating chloride ion homeostasis. However, sugars not only serve as energy sources and structural materials, but also help osmosis, which has an essential role in plant growth (Archetti et al., 2009; Dong et al., 2013). In addition, sugars also can function as signal molecules in different biological pathways in different plants species (Gazzarrini and Mccort, 2001; Solfanelli et al., 
Table 2. Functional annotation of the 18 enriched genes during the quantitative trait loci for red pigments in peach fruit flesh.

\begin{tabular}{lcccc}
\hline Gene accession & Chromosome & Transcript start (base pair) & Transcript stop (base pair) & Description \\
\hline ppa016440m & 1 & 11780460 & 11781407 & Sugar carrier protein C \\
ppa020244m & 1 & 11786475 & 11787125 & Sugar carrier protein C \\
ppa023512m & 1 & 11889517 & 11896074 & Chloride channel protein CLC-d \\
ppa004132m & 1 & 11903999 & 11909035 & Sugar transport protein 13 \\
ppa004417m & 1 & 11913147 & 11915173 & Sugar transport protein 13 \\
ppa004420m & 1 & 11915952 & 11917893 & Sugar transport protein 13 \\
ppa020009m & 1 & 11932661 & 11934856 & Carbohydrate transmembrane transporter \\
ppa004423m & 1 & 11973835 & 11975769 & Sugar transport protein 13 \\
ppa004424m & 1 & 11977117 & 11979137 & Sugar transport protein 13 \\
ppa004439m & 1 & 11991191 & 11994292 & Sugar transport protein 13 \\
ppa023527m & 1 & 11999707 & 12002134 & Sugar transport protein 13 \\
ppa008689m & 1 & 12995661 & 13000030 & Xyloglucan galactosyltransferase \\
ppa018375m & 1 & 13041816 & Xyloglucan galactosyltransferase \\
ppa007371m & 1 & 12997348 & 13049409 & Xyloglucan galactosyltransferase \\
ppa004844m & 1 & 13040229 & 13053908 & Xyloglucan galactosyltransferase \\
ppa005908m & 1 & 13047652 & 13079987 & Xyloglucan galactosyltransferase \\
ppa025271m & 1 & 13052324 & Xyloglucan galactosyltransferase \\
ppa006308m & 1 & 13079430 & 13083502 & Xyloglucan galactosyltransferase
\end{tabular}

${ }^{\mathrm{z}}$ Each accession name was annotated using peach reference genome v1.0 (Verde et al., 2013).

A
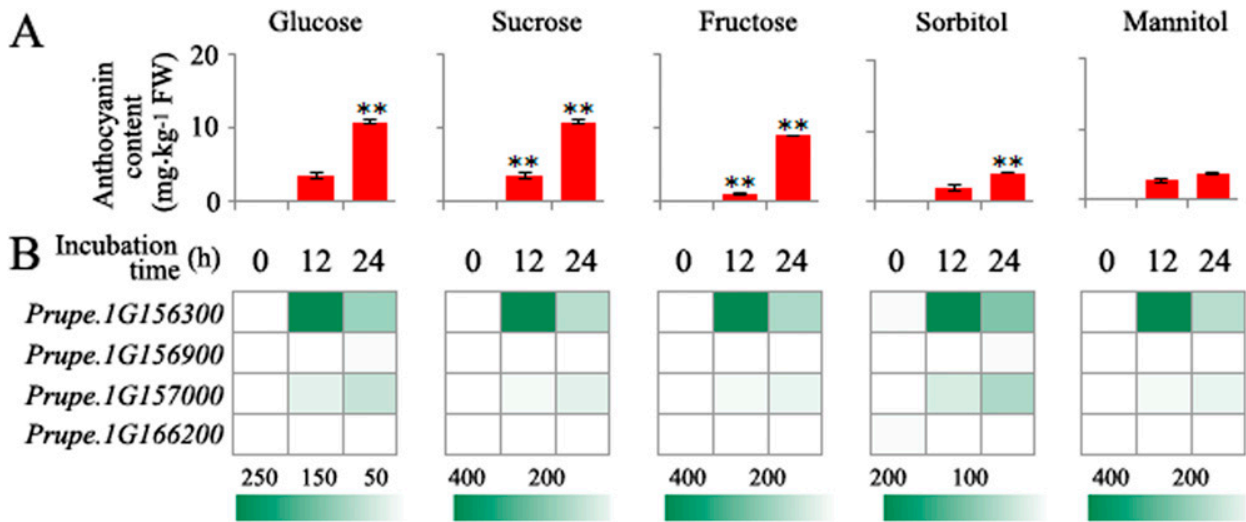

Fig. 4. (A) Anthocyanin content and (B) gene expression profile using RNA-seq in the fruit flesh of a red-fleshed peach 'Tianjin Shui Mi' induced by different sugar, including glucose, sucrose, fructose, and sorbitol, at 0,12 , and $24 \mathrm{~h}$, compared with the control (mannitol treatment). The anthocyanin content is expressed as the mean $\pm \mathrm{SD}$ of triplicates in each sample. Asterisks indicate significant differences between the sugar and the control in 12- or 24 -h treatment $(* * P<0.01$, one-way analysis of variance). $\mathrm{FW}=$ fresh weight.

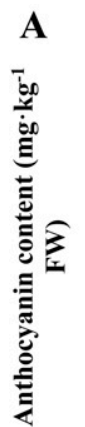

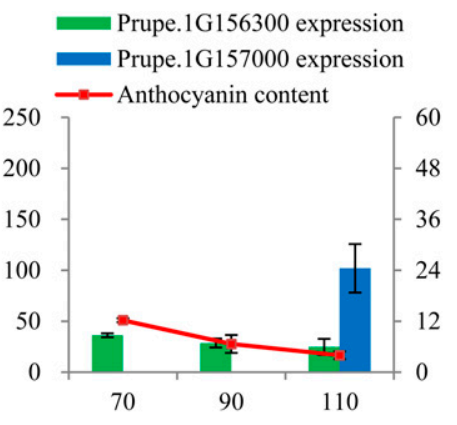

Time after full bloom (d)
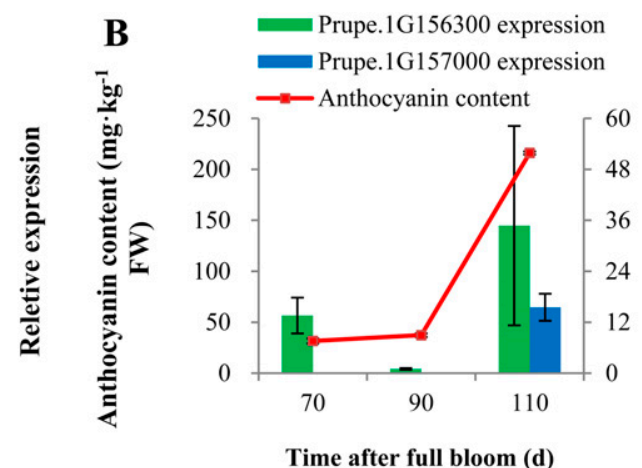

Fig. 5. The relative expression of different genes using quantitative real-time polymerase chain reaction and anthocyanin content in the fruit flesh during the development period of white-flesh [(A) 'Hakuho'] and red flesh [(B) 'Tianjin Shui Mi'] peach fruit. $\mathrm{FW}=$ fresh weight.
2006), and notably can enhance anthocyanin production in plants (Ai et al., 2016). Sucrose is involved in the expression of numerous genes related to anthocyanin biosynthesis, as well as in the regulation of transcription factors (Cheng and $\mathrm{Li}, 2010$ ), and can specifically induce the anthocyanin biosynthetic pathway in arabidopsis (Solfanelli et al., 2006). Similarly, exogenous glucose treatment has also been found to enhance anthocyanin content in the petals of Paeonia suffruticosa 'Luoyang Hong' cut flowers (Zhang et al., 2015). Moreover, these observations have been validated in Vitis vinifera cells (Gollop et al., 2001, 2002). Certain genes associated with sugar have been found to be enriched in the markers associated with anthocyanin content in peach (Nikkhah et al., 2007). Therefore, in the present study, we examined the effects of exogenous sugar treatment on fruit flesh to screen the 17 candidate genes. Our RNA-seq results indicated that among the 17 genes induced in fruit flesh; only three genes (Prupe.1G56300, Prupe.1G56900, and Prupe. 1G15700) showed an expression pattern similar to that of sugarinduced anthocyanin levels. When we further analyzed the expression of these three genes in fruit flesh during different periods of fruit development, we observed that ex- 
pression of Prupe.1G156300, a sugar transporter gene, was positively correlated with anthocyanin accumulation in redfleshed fruit; however, further analysis, including functional studies, are necessary to confirm the role of this candidate gene.

It was noticed that both mannitol and sorbitol increased anthocyanin content of cultured flesh (Fig. 4A), and these findings may be interpreted as the osmotic effects of these sugars on anthocyanin biosynthesis (Do and Cormier, 1990; Zhang et al., 2015).

\section{Conclusion}

To identify the candidate gene regulating anthocyanin content in peach, we performed a GWAS and candidate gene screening, and initially detected an association signal in the 11.7 to $13.1 \mathrm{Mb}$ region of $\mathrm{Chr}$. 1 . GO annotation of the 146 genes mapping to this region, indicated that 17 may be involved in sugar metabolism, and we accordingly focused on these in our subsequent analyses. Among these 17 genes, the expression of Prupe.1G156300, which encodes a sugar transporter, was found to be positively correlated with anthocyanin accumulation in fruit flesh of two red peach cultivars. These findings enabled us to provisionally characterize the genetic basis of anthocyanin biosynthesis in peach fruit.

\section{Literature Cited}

Ai, T.N., A.H. Naing, M. Arun, S.H. Lim, and C.K. Kim. 2016. Sucrose-induced anthocyanin accumulation in vegetative tissue of petunia plants requires anthocyanin regulatory transcription factors. Plant Sci. 252:144-150.

Akane, Y., T. Toshiko, O. Tomohiro, N. Naoki, K. Maiko, M. Kasumi, F. Toshifumi, M. Hayato, and K. Yoji. 2018. Single oral administration of anthocyanin rescues smoking-induced endothelial dysfunction in young smokers but sacilitates oxidative stress in non-smokers. Food Nutr. Sci. 9:179-190.

Allan, A.C., R.P. Hellens, and W.A. Laing. 2008. MYB transcription factors that colour our fruit. Trends Plant Sci. 13:99-102.

Archetti, M., T.F. Ring, S.B. Hagen, N.M. Hughes, S.R. Leather, D.W. Lee, S.L. Yadun, H.J. Ougham, P.G. Schaberg, and H. Thomas. 2009. Unravelling the evolution of autumn colours: An interdisciplinary approach. Trends Ecol. Evol. 24:166-173.

Beruter, J. and M.E. Studer Feusi. 1995. Comparison of sorbitol transport in excised tissue discs and cortex tissue of intact apple fruit. J. Plant Physiol. 146:95-102.

GitHub, Inc.. 2016. BGI-FlexLab. 1 Sept. 2016. <https://github.com/ BGI-flexlab/SOAPnuke/>.

Bradbury, P.J., Z. Zhang, D.E. Kroon, T.M. Casstevens, Y. Ramdoss, and E.S. Buckler. 2007. TASSEL: Software for association mapping of complex traits in diverse samples. Bioinformatics 23:2633-2635.

Cao, K., L.R. Wang, G.R. Zhu, W.C. Fang, C.W. Chen, and J. Luo. 2012. Genetic diversity, linkage disequilibrium, and association mapping analyses of peach (Prunus persica) landraces in China. Tree Genet. Genomes 8:975-990.

Cao, K., Z. Zhou, Q. Wang, J. Guo, P. Zhao, G.R. Zhu, W.C. Fang, X.W. Wang, X.L. Wang, Z.X. Tian, and L.R. Wang. 2016. Genome wide association study of 12 agronomic traits in peach. Nat. Commun. 7:13246.

Cao, K., T.Y. Ding, D.M. Mao, G.R. Zhu, W.C. Fang, C.W. Chen, X.W. Wang, and L.R. Wang. 2018. Transcriptome analysis reveals novel genes involved in anthocyanin biosynthesis in the flesh of peach. Plant Physiol. Biochem. 123:94-102.

Chalker, S.L. 2002. Do anthocyanins function as osmoregulators in leaf tissues. Adv. Bot. Res. 37:103-106.

Chalker, S.L. 2010. Environmental significance of anthocyanins in plant stress responses. Photochem. Photobiol. 70:1-9.
Chen, P.N., S.C. Chu, H.L. Chiou, W.H. Kuo, C.L. Chiang, and Y.S. Hsieh. 2006. Mulberry anthocyanins, cyaniding 3-rutinoside and cyaniding 3-glucoside, exhibited an inhibitory effect on the migration and invasion of a human lung cancer cell line. Cancer Lett. 235:248-259.

Cheng, H.Y. and D.H. Li. 2010. Studies on the synthesis and accumulation of anthocyanin in plants with light, sugar and hormones. Subtrop. Plant Sci. 39:82-86.

Choi, S.W., E.J. Chang, and T.Y. Ha. 1997. Antioxidative activity of acylate anthocyani isolated from fruit and vegetables. J. Food Sci. Nutr. 2:191-196.

Conesa, A. and S. Gotz. 2008. Blast2GO: A comprehensive suite for functional analysis in plant genomics. Intl. J. Plant Genomics 2008:1-12.

Diedhiou, C.J. and D. Golldack. 2006. Salt-dependent regulation of chloride channel transcripts in rice. Plant Sci. 170:793-800.

Ding, T.Y. 2017. The evaluation of polyphenol in peach and its QTL mapping. Chinese Acad. Agr. Sci., Beijing, MS Thesis.

Dirlewanger, E., E. Graziano, T. Joobeur, F. Garriga-Caldere, P. Cosson, W. Howad, and P. Arus. 2004. Comparative mapping and marker-assisted selection in Rosaceae fruit crops. Proc. Natl. Acad. Sci. USA 101:9891-9896.

Do, C.B. and F. Cormier. 1990. Accumulation of anthocyanins enhanced by a high osmotic potential in grape (Vitis vinifera L.) cell suspensions. Plant Cell Rpt. 9:143-146.

Dong, H.S., G.C. Myoung, K.L. Hyun, C. Misuk, B.C. Sang, C. Giltsu, and I.P. Youn. 2013. Calcium dependent sucrose uptake links sugar signaling to anthocyanin biosynthesis in Arabidopsis. Biochem. Biophys. Res. Commun. 430:634-639.

Donoso, J.M., R. Picañol, O. Serra, W. Howad, S. Alegre, P. Arús, and I. Eduardo. 2016. Exploring almond genetic variability useful for peach improvement: Mapping major genes and QTLs in two interspecific almond $\times$ peach populations. Mol. Breed. 36:16.

Eduardo, I., I. Pacheco, G. Chietera, D. Bassi, C. Pozzi, A. Vecchietti, and L. Rossini. 2011. QTL analysis of fruit quality traits in two peach intraspecific populations and importance of maturity date pleiotropic effect. Tree Genet. Genomes 7:323-335.

Forkmann, G. and S. Martens. 2001. Metabolic engineering and applications of flavonoids. Curr. Opin. Biotechnol. 12:155-160.

Frett, T.J., G.L. Reighard, W.R. Okie, and K. Gasic. 2014. Mapping quantitative trait loci associated with blush in peach [Prunus persica (L.) Batsch]. Tree Genet. Genomes 10:367-381.

Gazzarrini, S. and P. Mccourt. 2001. Genetic interactions between $\mathrm{ABA}$, ethylene and sugar signaling pathways. Curr. Opin. Plant Biol. 4:387-391.

Gollop, R., S. Even, T.V. Colova, and A. Perl. 2002. Expression of the grape dihydroflavonol reductase gene and analysis of its promoter region. J. Expt. Bot. 53:1397-1409.

Gollop, R., S. Farhi, and A. Perl. 2001. Regulation of the leucoanthocyanidin dioxygenase gene expression in Vitis vinifera. Plant Sci. 161:579-588.

Gould, K., K. Davies, and C. Winefield. 2009. Anthocyanins: Biosynthesis, functions, and applications. Springer-Verlag, Heidelberg, Germany.

Gyawali, S., M.L. Otte, S. Chao, J. Abderazzek, D.L. Jacob, R. Amezrou, and R.S. Verma. 2017. Genome wide association studies (GWAS) of element contents in grain with a special focus on zinc and iron in a world collection of barley (Hordeum vulgare). J. Cereal Sci. 77:266-274.

Jaakola, L. 2013. New insights into the regulation of anthocyanin biosynthesis in fruits. Trends Plant Sci. 18:477-483.

Jia, H.H. 2013. Roles and mechanisms of sucrose and jasmonic acid signals in the regulation of strawberry fruit development. China Agr. Sci. Univ., Beijing, PhD Diss.

Jiao, Y., R. Ma, Z. Shen, J. Yan, and M. Yu. 2014. Gene regulation of anthocyanin biosynthesis in two blood-flesh peach [Prunus persica (L.) Batsch] cultivars during fruit development. J. Zhejiang Univ. Sci. B 15:809-819.

Karageorgou, P. and Y. Manetas. 2006. The importance of being red when young: Anthocyanins and the protection of young leaves of Quercus coccifera from insect herbivory and excess light. Tree Physiol. 26:613-621. 
Kim, T.S., Q. He, K.W. Kim, M.Y. Yoon, W.H. Ra, F.P. Li, W. Tong, J. Yu, W.H. Oo, B. Choi, E.B. Heo, B.K. Yun, S.J. Kwon, S.W Kwon, Y.H. Cho, C.Y. Lee, B.S. Park, and Y.J. Park. 2016. Genomewide resequencing of krice-core reveals their potential for future breeding, as well as functional and evolutionary studies in the postgenomic era. BMC Genomics 17:408.

Korte, A. and A. Farlow. 2013. The advantages and limitations of trait analysis with GWAS: A review. Plant Methods 9:29.

Lila, M.A., B. Burtonfreeman, M. Grace, and W. Kalt. 2016. Unraveling anthocyanin bioavailability for human health. Annu. Rev. Food Sci. Technol. 7:375-393.

Lin-Wang, K., K. Bolitho, K. Grafton, A. Kortstee, S. Karunairetnam, T.K. McGhie, R.V. Espley, R.P. Hellens, and A.C. Allan. 2010. An R2R3 MYB transcription factor associated with regulation of the anthocyanin biosynthetic pathway in Rosaceae. BMC Plant Biol. 10:50.

Livak, K.J. and T.D. Schmittgen. 2001. Analysis of relative gene expression data using real-time quantitative PCR and the $2^{-\triangle \triangle \mathrm{CT}}$ method. Methods 25:402-408.

Nikkhah, E., M. Khayamy, R. Heidari, and R. Jamee. 2007. Effect of sugar treatment on stability of anthocyanin pigments in berries. J. Biol. Sci. 7:1412-1417.

Petroni, K. and C. Tonelli. 2011. Recent advances on the regulation of anthocyanin synthesis in reproductive organs. Plant Sci. 181:219-229. Quilot, B., B.H. Wu, J. Kervella, M. Génard, M. Foulongne, and K. Moreau. 2004. QTL analysis of quality traits in an advanced backcross between Prunus persica cultivars and the wild relative species P. davidiana. Theor. Appl. Genet. 109:884-897.

Rahim, M.A., N. Busatto, and L. Trainotti. 2014. Regulation of anthocyanin biosynthesis in peach fruits. Planta 240:913-929.

Revilla, P., V.M. Rodríguez, A. Ordás, R. Rincent, A. Charcosset, C. Giauffret, A.E. Melchinger, C.X. Chön, E. Bauer, T. Altmann, D. Brunel, J.M. González, L. Campo, M. Ouzunova, A. Álvarez, J.P. Galarreta, J. Laborde, and R.N. Malvar. 2016. Association mapping for cold tolerance in two large maize inbred panels. BMC Plant Biol. 16:127.

Shen, Z., C. Confolent, P. Lambert, J.L. Poessel, T.B. Quilot, M. Yu, and R. Ma. and Pascal T2013. Characterization and genetic mapping of a new blood-flesh trait controlled by the single dominant locus $D B F$ in peach. Tree Genet. Genomes 9:1435-1446.

Shulaev, V., S.S. Korban, B. Sosinski, A.G. Abbott, H.S. Aldwinckle, K.M. Folta, A. Iezzoni, D. Main, P. Arús, A.M. Dandekar, K. Lewers, S.K. Brown, T.M. Davis, S.E. Gardiner, D. Potter, and R.E. Veilleux. 2008. Multiple models for Rosaceae genomics. Amer. Soc. Plant Biol. 147:985-1003.

Solfanelli, C., A. Poggi, E. Loreti, A. Alpi, and P. Perata. 2006. Sucrose-specific induction of the anthocyanin biosynthetic pathway in Arabidopsis. Plant Physiol. 140:637-646.

Togninalli, M., Ü. Seren, D. Meng, J. Fitz, M. Nordborg, D. Weigel, K. Borgwardt, A. Korte, and G.D. Grimm. 2018. The AraGWAS Catalog: A curated and standardized Arabidopsis thaliana GWAS catalog. Nucleic Acids Res. 46:D1150-D1156.

Tomas-Barberan, F.A., M.I. Gil, P. Cremin, A.L. Waterhouse, B. HessPierce, and A.A. Kader. 2001. HPLC-DAD-ESIMS analysis of phenolic compounds in nectarines, peaches, and plums. J. Agr. Food Chem. 49:4748-4760.

Trapnell, C., B.A. Williams, G. Pertea, A. Mortazavi, G. Kwan, M.J. van Baren, S.L. Salzberg, B.J. Wold, and L. Pachter. 2010. Transcript assembly and quantification by RNA-Seq reveals unannotated transcripts and isoform switching during cell differentiation. Nat. Biotechnol. 28:511-515.

Tsukaya, H. 2004. Leaf shape: Genetic controls and environmental factors. Intl. J. Dev. Biol. 49:547-555.

Tuan, P.A., S. Bai, H. Yaegaki, T. Tamura, and K. Oda. 2015. The crucial role of PpMYB10.1 in anthocyanin accumulation in peach and relationships between its allelic type and skin color phenotype. BMC Plant Biol. 15:280.

Turner, S.D. 2014. qqman: An R package for visualizing GWAS results using Q-Q and manhattan plots. 30 Nov. 2017. <http://cran.rproject.org/web/packages/qqman/>.
Turuspekov, Y., A. Baibulatova, K. Yermekbayev, L. Tokhetova, V. Chudinov, G. Sereda, M. Ganal, S. Griffiths, and S. Abugalieva. 2017. GWAS for plant growth stages and yield components in spring wheat (Triticum aestivum L.) harvested in three regions of Kazakhstan. BMC Plant Biol. 17:190.

Verde, I., A.G. Abbott, S. Scalabrin, S. Jung, S. Shu, F. Marroni, T. Zhebentyayeva, M.T. Dettori, J. Grimwood, F. Cattonaro, A. Zuccolo, L. Rossini, J. Jenkins, E. Vendramin, L.A. Meisel, V. Decroocq, B. Sosinski, S. Prochnik, T. Mitros, A. Policriti, G. Cipriani, L. Dondini, S. Ficklin, D.M. Goodstein, P. Xuan, C. Del Fabbro, V. Aramini, D. Copetti, S. Gonzalez, D.S. Horner, R. Falchi, S. Lucas, E. Mica, J. Maldonado, B. Lazzari, D. Bielenberg, R. Pirona, M. Miculan, A. Barakat, R. Testolin, A. Stella, S. Tartarini, P. Tonutti, P. Arús, A. Orellana, C. Wells, D. Main, G. Vizzotto, H. Silva, F. Salamini, J. Schmutz, M. Morgante, and D.S. Rokhsar. 2013. The high-quality draft genome of peach (Prunus persica) identifies unique patterns of genetic diversity, domestication and genome evolution. Nat. Genet. 45:487-494.

Verde, I., J. Jenkins, L. Dondini, S. Micali, G. Pagliarani, E. Vendramin, R. Paris, V. Aramini, L. Gazza, L. Rossini, D. Bassi, M. Troggio, S.Q. Shu, J. Grimwood, S. Tartarini, M.T. Dettori, and J. Schmutz. 2017. The peach v2.0 release: High-resolution linkage mapping and deep resequencing improve chromosome-scale assembly and contiguity. BMC Genomics 18:225.

Wang, L.R. and G.R. Zhu. 2005. Descripters and data standard for peach. China Agr. Press, Beijing, China.

Wei, Q., Y. Liu, G. Zhou, Q. Li, C. Yang, and S. Peng. 2013. Overexpression of $C s C L C c$, a chloride channel gene from Poncirus trifoliata, enhances salt tolerance in Arabidopsis. Plant Mol. Biol. Rpt. 31:1548-1557.

Wen, L.N., X.W. Jiang, J.X. Sun, X. Li, X.S. Li, L.M. Tian, Y. Li, and W.B. Bai. 2018. Cyanidin-3-O-glucoside promotes the biosynthesis of progesterone through the protection of mitochondrial function in $\mathrm{Pb}$-exposed rat leydig cells. Food Chem. Toxicol. 112:427-434.

Xia, E.Q., G.F. Deng, Y.J. Guo, and H.B. Li. 2010. Biological activities of polyphenols from grapes. Intl. J. Mol. Sci. 11:622-646.

$\mathrm{Xu}, \mathrm{W}$., C. Dubos, and L. Lepiniec. 2015. Transcriptional control of flavonoid biosynthesis by MYB-bHLH-WDR complexes. Trends Plant Sci. 20:176-185.

Yang, J., S.H. Lee, M.E. Goddard, and P.M. Visscher. 2011. GCTA: A tool for genome-wide complex trait analysis. Amer. J. Hum. Genet. 88:76-82.

Yang, S.W., P. Tian, L.J. Li, D.T. Lu, L. Qiao, J.J. Zhao, L. Qiao, G. Chuan, J. Zheng, and J.C. Zhang. 2018. Extraction of anthocyanins from purple wheat and its effect on antioxidant activity and inhibitory on tumor cells. J. Agr. Sci. Technol. 20:1-8.

Ye, J., L. Fang, H.K. Zheng, Y. Zhang, J. Chen, Z.J. Zhang, J. Wang, S.T. Li, R.Q. Li, L. Bolund, and J. Wang. 2006. WEGO: A web tool for plotting GO annotations. Nucleic Acids Res. 34:293-297.

Zhang, C., J. Fu, Y. Wang, S. Gao, D. Du, F. Wu, J. Guo, and L.B. Dong. 2015. Glucose supply improves petal coloration and anthocyanin biosynthesis in Paeonia suffruticosa 'Luoyang Hong' cut flowers. Postharvest Biol. Technol. 101:73-81.

Zhang, G.H. 2008. Theories on the cultivation of fruit trees. China Agr. Press, Beijing, China.

Zhou, H., K. Lin-Wang, H. Wang, C. Gu, A.P. Dare, R.V. Espley, H. He, A.C. Allan, and Y. Han. 2015. Molecular genetics of bloodfleshed peach reveals activation of anthocyanin biosynthesis by $N A C$ transcription factors. Plant J. 82:105-121.

Zhou, L.J., Y.Y. Li, R.F. Zhang, C.L. Zhang, X.B. Xie, C. Zhao, and J.Y. Hao. 2017. The sumo E3 ligase mdsiz1 promotes anthocyanin accumulation by sumoylating $M d M Y B 1$ under low temperature conditions in apple. Plant Cell Environ. 40:2068-2080.

Zhu, H., R. Xia, B. Zhao, Y.Q. An, C.D. Dardick, A.M. Callahan, and Z.R. Liu. 2012. Unique expression, processing regulation, and regulatory network of peach (Prunus persica) miRNAs. BMC Plant Biol. 12:149. 

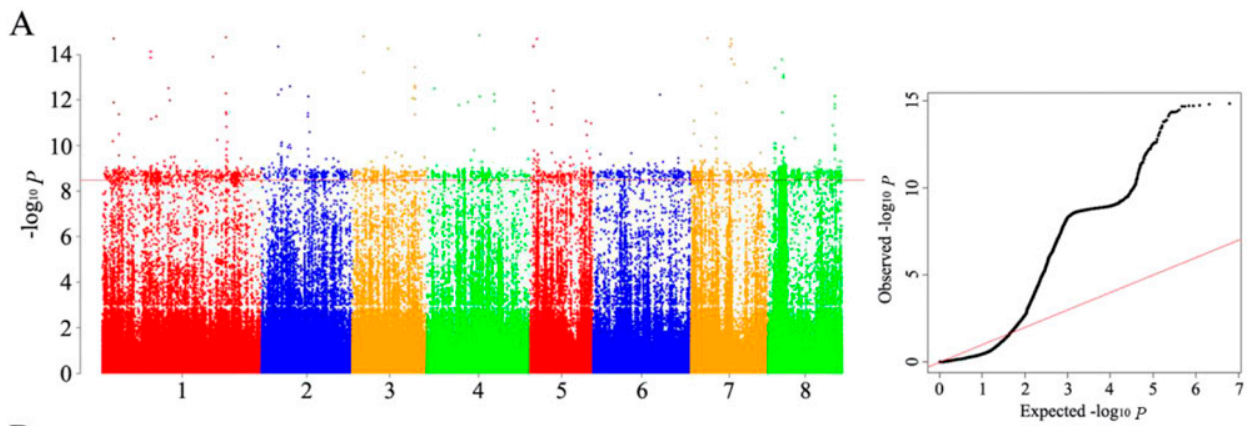

B
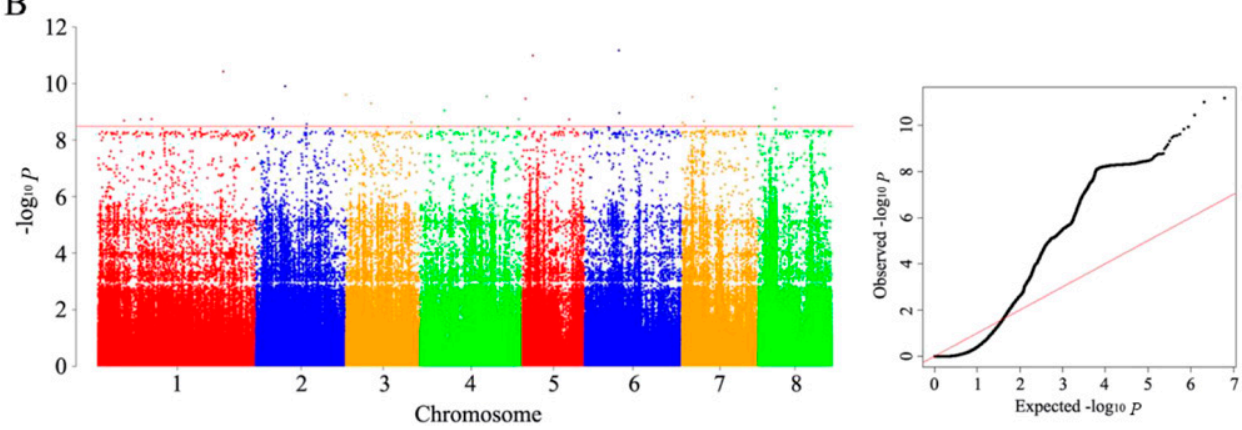

Supplemental Fig. 1. Manhattan and quantile-quantile plots of estimated $\log _{10} P$ from association analysis of cyanidin-3-glucoside content in 2014 (A) and 2015 (B) in fruit of 129 accessions using mixed linear model. Negative $\log _{10} P$ values from the genome-wide scan were plotted against single-nucleotide polymorphism position on each of the eight chromosomes. The dotted line indicates the genome-wide significance threshold $\left(\cdot \log _{10} P=8.49\right)$. 
Supplemental Table 1. Prunus samples used in this study and their key agronomic traits.

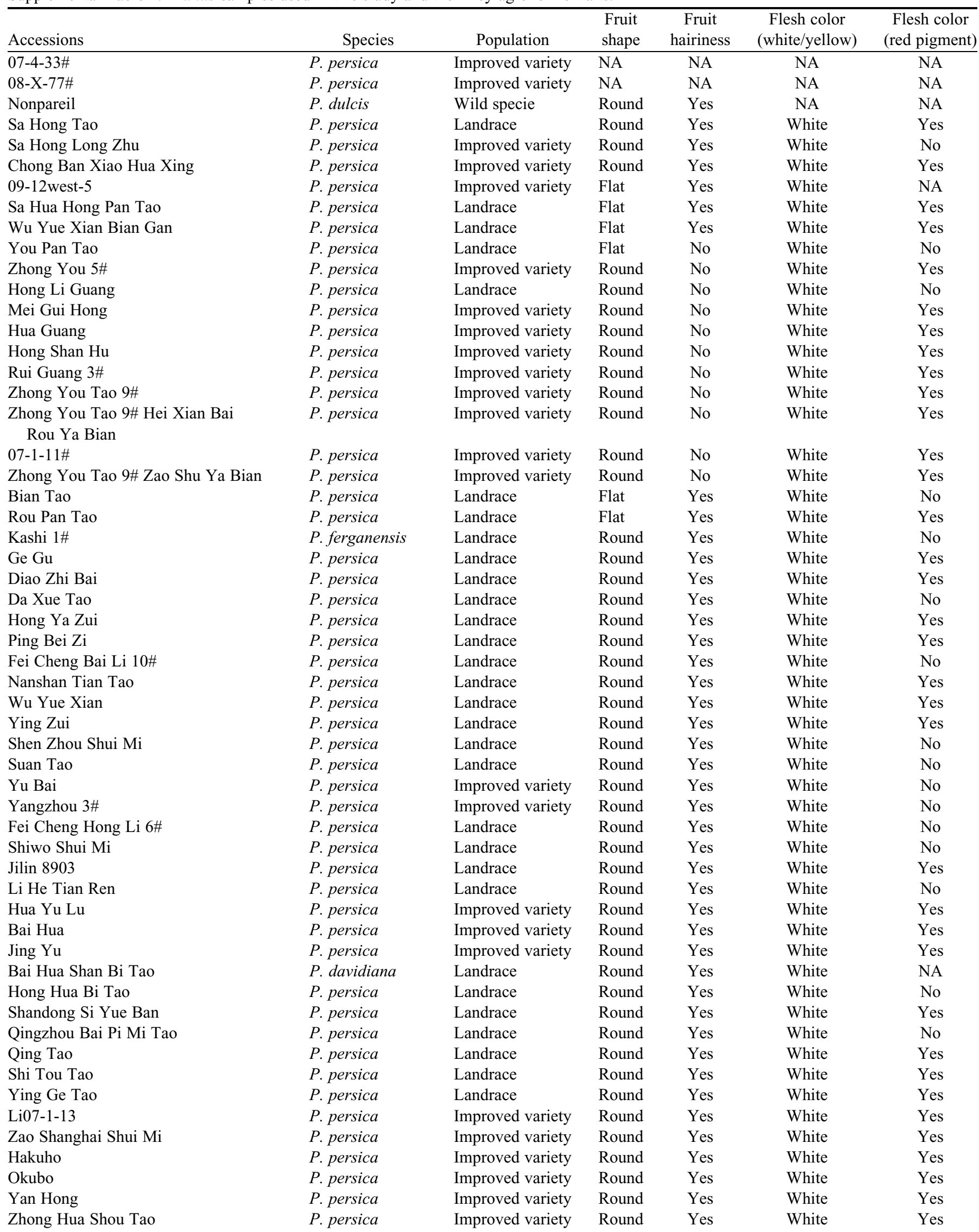


Supplemental Table 1. Continued.

\begin{tabular}{|c|c|c|c|c|c|c|}
\hline Accessions & Species & Population & $\begin{array}{l}\text { Fruit } \\
\text { shape }\end{array}$ & $\begin{array}{c}\text { Fruit } \\
\text { hairiness }\end{array}$ & $\begin{array}{c}\text { Flesh color } \\
\text { (white/yellow) }\end{array}$ & $\begin{array}{l}\text { Flesh color } \\
\text { (red pigment) }\end{array}$ \\
\hline $07-2-24 \#$ & P. persica & Improved variety & Round & Yes & White & Yes \\
\hline Hong Hua Shan Tao & P. davidiana & Wild specie & Round & Yes & White & No \\
\hline Guang He Tao (Rikaze) & P. mira & Wild specie & Round & Yes & White & No \\
\hline Guang He Tao (Aba) & P. mira & Wild specie & Round & Yes & White & No \\
\hline Bi Nan I & P. persica & Landrace & Round & Yes & White & Yes \\
\hline Shuang Xi Hong & P. persica & Improved variety & Round & No & Yellow & Yes \\
\hline Shu Guang Hong Xian Ya Bian & P. persica & Improved variety & Round & No & Yellow & Yes \\
\hline Hei Bu Dai & P. persica & Landrace & Round & Yes & White & Yes \\
\hline Tian Jin Shui Mi & P. persica & Landrace & Round & Yes & White & Yes \\
\hline Wuhan 2\# & P. persica & Improved variety & Round & Yes & White & Yes \\
\hline Harrow Blood & P. persica & Improved variety & Round & Yes & White & Yes \\
\hline Huang Jin Pan Tao & P. persica & Landrace & Flat & Yes & Yellow & No \\
\hline Zhong You Pan 2\# & P. persica & Improved variety & Flat & No & Yellow & No \\
\hline Zhong You Pan 4\# & P. persica & Improved variety & Flat & No & Yellow & Yes \\
\hline Ping Ding You Pan Tao & P. persica & Improved variety & Flat & No & Yellow & No \\
\hline Rui Guang 2\# & P. persica & Improved variety & Round & No & Yellow & No \\
\hline NJN76 & P. persica & Improved variety & Round & No & Yellow & Yes \\
\hline Okitsu & P. persica & Improved variety & Round & No & Yellow & Yes \\
\hline Legrand & P. persica & Improved variety & Round & No & Yellow & Yes \\
\hline May Fire & P. persica & Improved variety & Round & No & Yellow & No \\
\hline Zhong You Tao 4\# & P. persica & Improved variety & Round & No & Yellow & Yes \\
\hline Kashi Huang Rou Li Guang & P. persica & Landrace & Round & No & Yellow & Yes \\
\hline Zhong You Tao 9\# Wan Shu Ya Bian & P. persica & Improved variety & Round & No & Yellow & Yes \\
\hline Zao Huang Pan Tao & P. persica & Improved variety & Flat & Yes & Yellow & Yes \\
\hline Mai Huang Pan Taao & P. persica & Improved variety & Flat & Yes & Yellow & Yes \\
\hline Da Lian 4-35 & P. persica & Improved variety & Flat & Yes & Yellow & Yes \\
\hline Xinjiang Huang Rou & P. ferganensis & Landrace & Round & Yes & Yellow & No \\
\hline Zhao Shu Huang Gan & P. persica & Landrace & Round & Yes & Yellow & Yes \\
\hline Elberta & P. persica & Improved variety & Round & Yes & Yellow & Yes \\
\hline Qing Si Tao & P. persica & Landrace & Round & Yes & Yellow & No \\
\hline Huo Lian Jin Dan & P. persica & Landrace & Round & Yes & Yellow & Yes \\
\hline Jin Feng & P. persica & Improved variety & Round & Yes & Yellow & Yes \\
\hline NJC77 & P. persica & Improved variety & Round & Yes & Yellow & No \\
\hline Maria Serena & P. persica & Improved variety & Round & Yes & Yellow & No \\
\hline Redhaven & P. persica & Improved variety & Round & Yes & Yellow & Yes \\
\hline Phillips & P. persica & Improved variety & Round & Yes & Yellow & No \\
\hline Mao Таo 2-1-55 & P. persica & Improved variety & Round & Yes & Yellow & Yes \\
\hline Xiamiao $1 \#$ & P. persica & Landrace & Round & Yes & Yellow & Yes \\
\hline Long 1-2-4 & P. persica & Landrace & Round & Yes & Yellow & Yes \\
\hline Zhu Fen Chui Zhi & P. persica & Landrace & Round & Yes & White & No \\
\hline Hong Shou Xing & P. persica & Landrace & Round & Yes & White & No \\
\hline Bai Gen Gan Su Tao & P. kansuensis & Wild specie & Round & Yes & White & No \\
\hline Hong Gen Gan Su Tao & P. kansuensis & Wild specie & Round & Yes & White & No \\
\hline
\end{tabular}


Supplemental Table 1. Continued.

\begin{tabular}{|c|c|c|c|c|c|c|}
\hline Accessions & Species & Population & $\begin{array}{l}\text { Fruit } \\
\text { shape }\end{array}$ & $\begin{array}{c}\text { Fruit } \\
\text { hairiness }\end{array}$ & $\begin{array}{c}\text { Flesh color } \\
\text { (white/yellow) }\end{array}$ & $\begin{array}{l}\text { Flesh color } \\
\text { (red pigment) }\end{array}$ \\
\hline Xinjiang Pan Tao & P. ferganensis & Landrace & Flat & Yes & White & No \\
\hline Xinjiang Pan Tao(pollen sterility) & P. ferganensis & Wild specie & Flat & Yes & White & No \\
\hline Hong Ye Tao & P. persica & Landrace & Round & Yes & White & No \\
\hline Hong Ye Tao Ya Bian & P. persica & Landrace & Round & Yes & White & No \\
\hline Hun Chun Tao & P. persica & Landrace & Round & Yes & White & Yes \\
\hline Xian Tao & P. persica & Landrace & Round & Yes & White & No \\
\hline Yuan Yang Chui Zhi & P. persica & Landrace & Round & Yes & White & No \\
\hline Fen Shou Xing & P. persica & Landrace & Round & Yes & White & Yes \\
\hline Hong Chui Zhi & P. persica & Landrace & Round & Yes & White & No \\
\hline Bailey & P. persica & Improved variety & Round & Yes & White & No \\
\hline Zhou Xing Shan Tao & P. davidiana & Wild specie & Round & Yes & Yellow & No \\
\hline Bai Wu 8\# & $P$. persica & Improved variety & Round & Yes & White & Yes \\
\hline
\end{tabular}

Note: NA indicates the data were absent. 